INPLASY

PROTOCOL

To cite: Cheng et al.

Comparison of the clinical effects and safety of normal saline and balanced

crystalloids in patients with diabetic ketoacidosis: a metaanalysis. Inplasy protocol

2021120014. doi:

10.37766/inplasy2021.12.0014

Received: 02 December 2021

Published: 02 December 2021

Corresponding author:

Shuping Cheng

chengsong199407@126.com

Author Affiliation:

Emergency Center, The

Second Affiliated Hospital of

Nanchang University.

Support: None.

Review Stage at time of this submission: Formal screening of search results against

eligibility criteria.

Conflicts of interest:

None declared.

\section{Comparison of the clinical effects and safety of normal saline and balanced crystalloids in patients with diabetic ketoacidosis: a meta-analysis}

Cheng, SP1; Wu, LD2; Liu, YF3; Deng, CX4.

Review question / Objective: Rehydration to correct internal environmental disorders is the cornerstone of the treatment of diabetic ketoacidosis (DKA), and the type of fluid used is still controversial. The purpose of this study is to evaluate the clinical effect and safety of balanced crystalloids and normal saline on DKA treatment.

Condition being studied: Normal saline has always been the traditional fluid choice for DKA fluid resuscitation. However, large amounts of saline may not be safe, which can lead to hyperchloremia and increase the risk of acute kidney injury (AKI) and acidosis associated with chloride levels. Therefore, in order to reduce the potential risks brought by normal saline, it has been proposed to use balanced crystalloids instead of normal saline to treat DKA. Previous studies have shown that balanced crystalloids (Ringer's lactate, Hartmann's solution, Plasma-Lyte, etc.) can reduce complications such as AKI and acidosis during fluid resuscitation in emergency and intensive care units. At present, the choice of fluid therapy for DKA patients is still inconclusive. The purpose of this meta-analysis is to compare the clinical efficacy and safety of normal saline and balanced crystalloids for DKA fluid resuscitation.

INPLASY registration number: This protocol was registered with the International Platform of Registered Systematic Review and Meta-Analysis Protocols (INPLASY) on 02 December 2021 and was last updated on 02 December 2021 (registration number INPLASY2021120014).

\section{INTRODUCTION}

Review question / Objective: Rehydration to correct internal environmental disorders is the cornerstone of the treatment of diabetic ketoacidosis (DKA), and the type of fluid used is still controversial. The purpose of this study is to evaluate the clinical effect and safety of balanced 
crystalloids and normal saline on DKA treatment.

Condition being studied: Normal saline has always been the traditional fluid choice for DKA fluid resuscitation. However, large amounts of saline may not be safe, which can lead to hyperchloremia and increase the risk of acute kidney injury (AKI) and acidosis associated with chloride levels. Therefore, in order to reduce the potential risks brought by normal saline, it has been proposed to use balanced crystalloids instead of normal saline to treat DKA. Previous studies have shown that balanced crystalloids (Ringer's lactate, Hartmann's solution, Plasma-Lyte, etc.) can reduce complications such as AKI and acidosis during fluid resuscitation in emergency and intensive care units. At present, the choice of fluid therapy for DKA patients is still inconclusive. The purpose of this metaanalysis is to compare the clinical efficacy and safety of normal saline and balanced crystalloids for DKA fluid resuscitation.

\section{METHODS}

Search strategy: Use the following keywords: (1) "diabetes" OR "diabetic ketoacidosis" OR "diabetic hyperglycemia emergency" OR "DKA"; (2) "normal saline" OR "saline" OR "0.9\%NS"; (3) "Balanced saline" OR "Balanced crystals" OR "Sodium lactate Ringer's solution" OR "Hartmann's solution" OR "Plasma-Lyte". Keywords use the Boolean calculation "and" combination to search. The literature search does not limit the language of publication.

Participant or population: Patients diagnosed with diabetic ketoacidosis (diagnostic criteria refer to guidelines of the American Diabetes Association in 2009).

Intervention: Balanced crystalloids(Ringer's lactate, Hartmann's solution and PlasmaLyte, etc.)

Comparator: Normal saline.
Study designs to be included: Randomized controlled trial, Prospective cohort study, Retrospective cohort study.

Eligibility criteria: (1) Randomized controlled trials or cohort studies (including retrospective cohort studies and prospective cohort studies) on the effect of normal saline and balanced crystals on DKA fluid resuscitation (2) No age limit; (3) No language limit for publication. The following studies were excluded: (1) review articles and case reports; (2) literature with no data available.

Information sources: We will search the following databases (The retrieval time is from the establishment of each database to November 2021.): China Knowledge Network Database (CNKI), WanFang Database (WanFang database), PubMed, Embase, and Cochrane Library.

Main outcome(s): DKA resolution time; The mean postresuscitation chloride; The mean postresuscitation bicarbonate

Additional outcome(s): Complications.

Data management: We recorded all the original data in the form of an electronic version.

Quality assessment / Risk of bias analysis: The research quality of randomized controlled studies is assessed by the risk of bias table in the Review Manager 5.3 tutorial; The cohort study used the Newcastle-Ottawa Scale (NOS) for literature quality assessment. The included studies will be evaluated by two researchers, and any differences could be resolved through discussion. If no agreement is reached through discussion and consultation, The decision should be made by a vote of the third researcher.

Strategy of data synthesis: RevMan $\mathbf{5 . 3}$ provided by the Cochrane Collaboration was used for data analysis. For continuous results, weighted mean differences (WMD) and $95 \%$ confidence interval $(\mathrm{Cl})$ were used, and Relative risk (RR) will be used for 
dichotomy results with $95 \%$ confidence interval $(\mathrm{Cl})$, The choice of random effect model or fixed effect model depends on the heterogeneity of the original research. In this study, the Cochrane $Q$ test will be used to analyze the heterogeneity between studies, If there was no significant difference by the $Q$ test $(P>0.10,12 \leq 50 \%)$, the fixed-effects model was used for the meta-analysis. Otherwise, we will choose sensitivity analysis, subgroup analysis or meta regression to explore the causes of heterogeneity.

Subgroup analysis: Perform subgroup analysis based on age.

Sensitivity analysis: If there was a significant difference ( $P \leq 0.10$ and $12>50 \%$ ), sensitivity analysis was conducted to find out the source of heterogeneity. Judge by the method of excluding research one by one, so as to test the robustness of the comprehensive results.

Language: No limitation of language.

Country(ies) involved: China.

Other relevant information: None.

Keywords: normal saline, balanced crystalloids, diabetic ketoacidosis.

Contributions of each author:

Author 1 - Shuping Cheng - The author designed the experimental plan, collect the data, analyze the data and drafted the manuscript.

Email: chengsong199407@126.com

Author 2 - Lidong Wu - The author read, provided feedback and approved the final manuscript.

Email: dongguawu@163.com

Author 3 - Yuanfei Liu - The author contributed to the selection of the literature.

Email: 113434597@qq.com

Author 4 - Chunxia Deng - The author contributed to the selection of the literature.

Email: dengchunxia@whu.edu.cn 